\title{
PRÁTICA E DURAÇÃO DO ALEITAMENTO MATERNO DE CRIANÇAS MATRICULADAS EM ESCOLAS PARTICULARES DO MUNICÍPIO DE SÃO PAULO, SP
}

\section{PRACTICE AND DURATION OF BREASTFEEDING OF CHILDREN REGISTERED IN PRIVATE SCHOOLS IN SÃO PAULO, SP}

\author{
Viviane Gabriela Nascimento Simon ${ }^{1}$ \\ José Maria Pacheco de Souza ${ }^{2}$ \\ Cláudio Leone ${ }^{3}$ \\ Sonia Buongermino de Souza ${ }^{4}$
}

Simon VGN et al. Prática e duração do aleitamento materno de crianças matriculadas em escolas particulares do município de São Paulo, SP. Rev Bras Crescimento Desenvolv Hum. 2009; 19(3): 393-402.

\section{Resumo:}

O presente estudo descreve a prática de aleitamento materno e verifica possíveis fatores de associação com a duração do aleitamento materno exclusivo e aleitamento materno em crianças de escolas particulares do município de São Paulo. Fizeram parte do estudo 566 crianças, com 2 a 6 anos completos de idade. Foi considerada como variável dependente a duração do aleitamento materno (aleitamento materno exclusivo e aleitamento materno), e como independente idade e escolaridade materna, condição de trabalho da mãe e sexo da criança. A caracterização da amostra é apresentada por meio de distribuições de freqüências. A variável aleitamento materno foi descrita em categorias, e para análise foi utilizada como variável contínua. Para a análise da relação entre duração do aleitamento materno e as variáveis independentes utilizou-se a técnica de regressão múltipla de Cox adotando-se critério $\mathrm{p}<0,05$ para decisão de significância. Não houve associação entre as variáveis estudadas e tempo de duração das duas formas de aleitamento. Cerca de $80 \%$ das crianças deixaram de ser amamentadas exclusivamente antes dos seis meses de vida, o que mostra a necessidade de continuar o desenvolvimento de ações para incentivo e apoio à amamentação.

Palavras-chaves: aleitamento materno exclusivo; pré-escolar; aleitamento materno; desmame.

1 Pesquisadora em nível de Pós-Doutorado do Departamento de Saúde Materno-Infantil. Faculdade de Saúde Pública. Universidade de São Paulo. São Paulo, SP, Brasil.

2 Professor Titular do Departamento de Epidemiologia da Faculdade de Saúde Pública da Universidade de São Paulo. São Paulo, SP, Brasil.

3 Professor titular do Departamento de Saúde Materno-Infantil. Faculdade de Saúde Pública. Universidade de São Paulo. São Paulo, SP, Brasil.

4 Professora Doutora do Departamento de Nutrição da Faculdade de Saúde Pública da Universidade de São Paulo. São Paulo, SP, Brasil.

Endereço para correspondência: Viviane Gabriela Nascimento Simon - Faculdade de Saúde Pública da Universidade de São Paulo - Departamento de Saúde Materno Infantil - Av. Dr. Arnaldo, 715 - 01246-904. São Paulo, SP-Brasil.

E-mail para contato: vivianesimon@usp.br

Instituição: Faculdade de Saúde Pública - Universidade de São Paulo - Declaração de conflito de interesse: Nada a Declarar Fonte financiadora: sem financiamento. - Palavras: texto 1814; resumo 187, abstract 173; tabelas 2; figuras 3; referências 18. Artigo baseado na tese de doutorado de VGN Simon, apresentada a Faculdade de Saúde Publica da Universidade de São Paulo, em 2007, intitulada "Relação entre aleitamento materno, alimentação complementar e sobrepeso e obesidade em crianças de 2 a 6 anos de idade matriculadas em escolas particulares no Município de São Paulo”. 


\begin{abstract}
:
The present study describes the practice of breast feeding and verifies possible associated factors with the duration of breast feeding in children from private schools of the city of São Paulo. The study was made with 566 children from 2 to 6 years of age. For the dependent variable, it was considered the duration of breast feeding (exclusive breast feeding and breast feeding), and as the independent variables maternal age and schooling, mother working condition, and sex of the child. The characterization of the sample is presented through frequency distributions. The variable breast feeding was described in categories, and for the analysis was used as a continuous variable. For the analysis of the relationship of breast feeding and the independent variables, the multiple regression techniques of Cox were used adopting $\mathrm{p}<0.05$ criterion for the decision significance. There was no association between the studied variables and the two forms of breast feeding. Approximately, $80 \%$ of children are no longer breast fed exclusively before six months of life, which shows the need to continue the development of actions to encourage and support breast feeding.
\end{abstract}

Key words: exclusive breast feeding; pre-school; breast feeding; weaning.

\section{INTRODUÇÃO}

O leite humano oferece os nutrientes de que a criança necessita para iniciar uma vida saudável, sendo essencial para o lactente até o sexto mês de vida, como alimento exclusivo ${ }^{1,2}$, representando forma natural de alimentar a criança, propiciando crescimento e desenvolvimento adequados. ${ }^{3}$

Nas últimas três décadas aconteceram avanços importantes para a prática do aleitamento materno no Brasil. Pesquisas populacionais mostraram que a duração mediana do aleitamento materno aumentou de 2,5 para 5,5 meses entre os anos de 1975 e $1989 .{ }^{4}$ Em 1992, a duração mediana foi de 4,5 meses $^{5}$ e em 1996 foi de 7 meses. ${ }^{6}$

Apesar do incentivo ao aleitamento materno exclusivo, o Brasil não conseguiu atingir a recomendação da Organização Mundial da Saúde ${ }^{1}$ e a mediana de aleitamento materno exclusivo em 1999 foi de 33,7 dias no país.? Estudos brasileiros mais recentes mostram mediana de aleitamento materno exclusivo de 1,5 e 2 meses e de aleitamento materno 10, 9 e 6,4 meses. ${ }^{8,9}$

Segundo o relatório do Ministério da Saúde (2009) ${ }^{10}$ a prevalência do aleitamento materno exclusivo em menores de 6 meses foi de $41 \%$ no conjunto das capitais brasileiras, sendo que a região Norte apresentou maior prevalência (45,9\%), seguida da Centro-Oeste $(45,0 \%)$, Sul $(43,9 \%)$ e Sudeste $(39,4 \%)$, com a região Nordeste apresentando a pior situação (37,0\%). A prevalência do aleitamento materno em crianças de 9 a 12 meses foi de $58,7 \%$ no conjunto das capitais brasileiras e Distrito Federal, sendo que, a região Norte apresentou a melhor situação (76,9\%), seguida do Centro-Oeste (64,1\%), Nordeste (59,1\%) e Sudeste $(51,4 \%)$. Com relação a esse indicador, a pior situação é a da região Sul (49,5\%).

Alguns estudos apontam fatores associados de forma positiva ao aleitamento materno exclusivo, como maior escolaridade materna, situação conjugal com vínculo, recém-nascido com idade gestacional maior de 37 semanas, mães com experiência anterior com amamentação e mulheres que residem em casa própria. ${ }^{11,6}$

Outros mostram que a interrupção precoce da amamentação exclusiva está associada com baixa renda familiar, pouca idade materna, primiparidade e retorno da mãe ao trabalho. ${ }^{12}$ 
O presente estudo descreve a prática de aleitamento materno e verifica possíveis fatores de associação com a duração do aleitamento materno exclusivo e aleitamento materno em crianças de escolas particulares do município de São Paulo.

\section{MÉTODO}

Estudo transversal, realizado com crianças de 2 a 6 anos de idade completos, matriculadas em escolas particulares, na Zona Norte do município de São Paulo.

Optou-se por estudar a faixa etária de pré-escolar, a fim de diminuir possível viés de memória da mãe, em relação à variável aleitamento materno. Para maior facilidade e operacionalidade no desenvolvimento da pesquisa, foi realizado contato telefônico com sete escolas particulares, que concordaram em participar do estudo.

O período de coleta de dados foi de agosto a novembro de 2004, e de março a maio de 2005. Devido à dificuldade da presença da mãe e/ou responsável durante o período de aula da criança, os formulários com instruções para o auto-preenchimento foram entregues pela escola para serem respondidos pelas próprias mães e/ou responsáveis e devolvidos, posteriormente, na escola. Foram entregues 809 formulários, sendo que foram devolvidos $70 \%$. Todos os formulários foram analisados após serem devolvidos, e para informações incompletas ou não compreendidas, utilizou-se contato telefônico diretamente com a mãe e/ou responsável, não havendo perdas nesta fase da pesquisa. Fizeram parte do estudo 566 crianças.

Na análise dos dados, foi considerada como variável dependente a duração do aleitamento materno, sendo considerados o aleitamento materno exclusivo (AME) e o aleitamento materno (AM), verificando até que idade, em meses, as crianças mamaram exclusivamen- te ao seio e até que idade foram amamentadas, respectivamente.

Para AME foram consideradas crianças que receberam somente leite materno, diretamente ou extraído, e nenhum outro liquido ou sólido, com exceção de gotas ou xaropes de vitaminas, minerais e/ou medicamentos, e para AM quando recebiam leite materno diretamente do seio ou extraído, independente de estar recebendo qualquer alimento liquido, incluindo leite não humano, conforme recomendação da Organização Mundial de Saúde. ${ }^{2}$

As variáveis independentes (explanatórias) foram: idade materna ( $\leq 35$ anos e $>35$ anos), escolaridade materna (ensino fundamental + ensino médio e ensino superior), condição de trabalho materno (trabalha fora e não trabalha) e sexo da criança. Para a análise, decidiu-se utilizar as variáveis de forma dicotômica; para a idade materna, as categorias foram obtidas adotando-se a mediana como ponto de corte.

A caracterização da amostra é apresentada por meio de distribuições de freqüências. A variável aleitamento materno foi descrita em categorias, e para análise foi utilizada como variável contínua. Para a análise da relação entre duração do aleitamento materno e as variáveis independentes utilizou-se a técnica de regressão múltipla de Cox adotando-se critério $\mathrm{p}<0,05$ para decisão de significância.

São apresentados os p descritivos e os intervalos com 95\% de confiança. O banco de dados foi elaborado utilizando-se a planilha Microsoft Excel 2002 e as análises estatísticas foram feitas utilizando-se o pacote Stata 10.1.

Este estudo é parte da pesquisa intitulada "Relação do aleitamento materno, alimentação complementar e obesidade e sobrepeso em crianças de 2 a 6 anos de idade matriculadas em escolas particulares no Município de São Paulo”. Todas as mães e/ou responsáveis pelas crianças que concordaram com a participação na pesquisa assinaram um Termo de Consentimento Livre e Esclarecido. O presente estudo 
foi aprovado pelo Comitê de Ética em Pesquisa da Faculdade de Saúde Pública da Universidade de São Paulo. (protocolo de aprovação $\mathrm{n}^{\circ} 1222$ - fevereiro de 2005).

\section{RESULTADOS}

A tabela 1 mostra que 78,6\% das crianças estiveram em aleitamento materno exclu- sivo por período menor que 6 meses de vida, e $24,1 \%$ permaneceram em aleitamento materno por um ano ou mais. Observa-se, também, que a maioria das mães tinha idade menor ou igual a 35 anos, trabalhava fora e tinha nível superior de escolaridade. Em relação ao sexo da criança, observa-se distribuição homogênea.

Tabela 1: Distribuição da amostra, segundo aleitamento materno, variáveis maternas e sexo da criança. São Paulo, SP, 2004/2005.

\begin{tabular}{|c|c|c|}
\hline Variáveis & Número de crianças & $\%$ \\
\hline \multicolumn{3}{|l|}{ AME (meses) } \\
\hline$<6$ & 445 & 78,6 \\
\hline$\geq 6$ & 121 & 21,4 \\
\hline \multicolumn{3}{|l|}{$\overline{\mathrm{AM} \text { (meses) }}$} \\
\hline $0-6$ & 191 & 33,7 \\
\hline $6-12$ & 239 & 42,2 \\
\hline 12 e mais & 136 & 24,1 \\
\hline \multicolumn{3}{|l|}{ Trabalho materno } \\
\hline Trabalha fora & 416 & 73,5 \\
\hline Não trabalha & 150 & 26,5 \\
\hline \multicolumn{3}{|l|}{ Escolaridade materna } \\
\hline Ensino Fundamental + Médio & 87 & 15,4 \\
\hline Ensino Superior & 479 & 84,6 \\
\hline \multicolumn{3}{|l|}{ Idade materna } \\
\hline$\leq 35$ anos & 299 & 52,8 \\
\hline$>35$ anos & 267 & 47,2 \\
\hline \multicolumn{3}{|l|}{ Sexo da criança } \\
\hline Masculino & 285 & 50,3 \\
\hline Feminino & 281 & 49,7 \\
\hline Total de crianças: & 566 & $100 \%$ \\
\hline
\end{tabular}


Figura 1: Proporção de crianças ainda em aleitamento materno exclusivo ou em aleitamento materno. São Paulo, SP, 2004/2005.

Proporção de crianças ainda em aleitamento materno exclusivo e proporção ainda em aleitamento materno

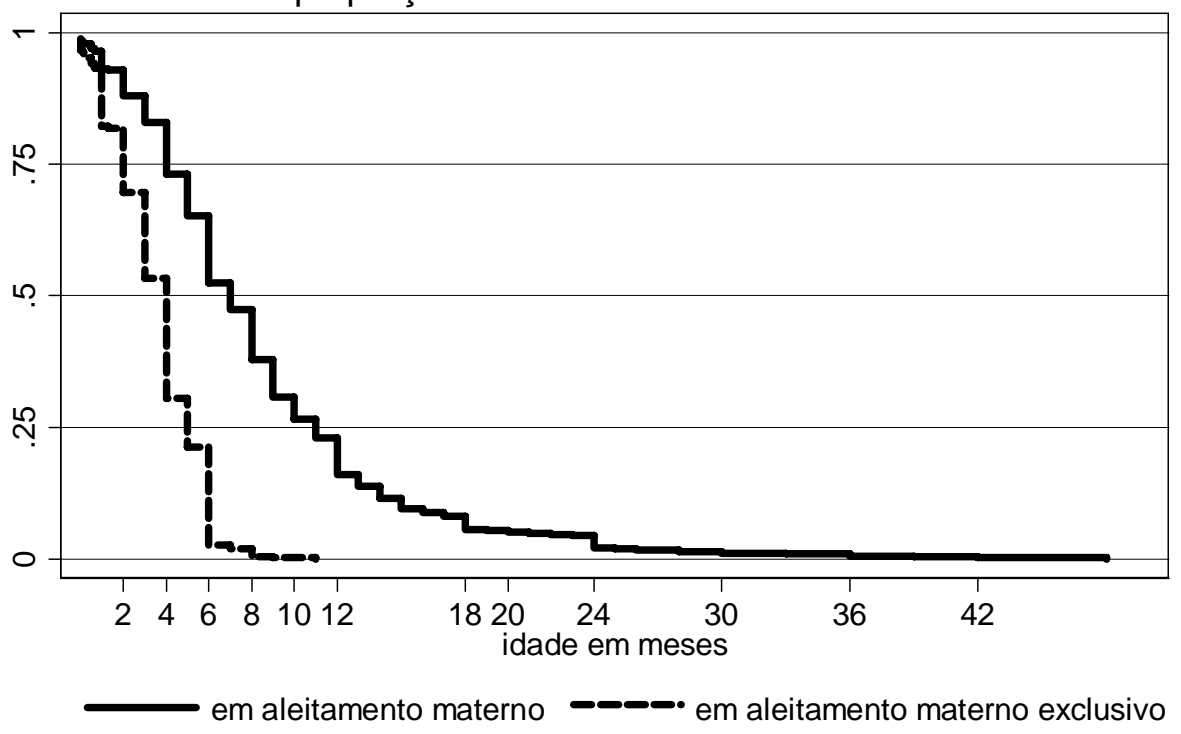

Figura 2: Proporção de crianças ainda em aleitamento materno exclusivo, segundo categorias de variáveis. São Paulo, SP, 2004/2005

Proporção de crianças ainda em aleitamento exclusivo, segundo categorias de variáveis
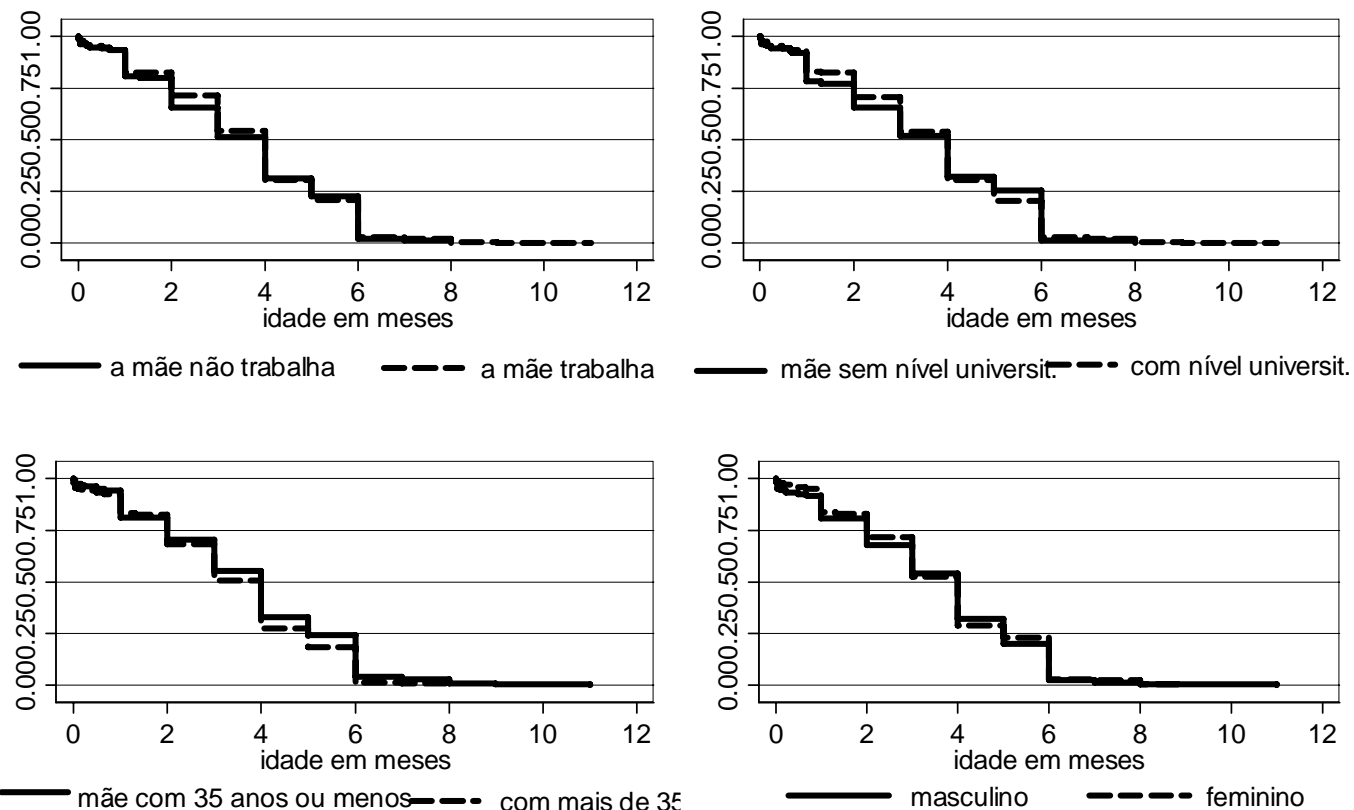
Figura 3: Proporção de crianças ainda em aleitamento materno, segundo categorias de variáveis. São Paulo, SP, 2004/2005.

\section{Proporção de crianças ainda em aleitamento, segundo categorias de variáveis}
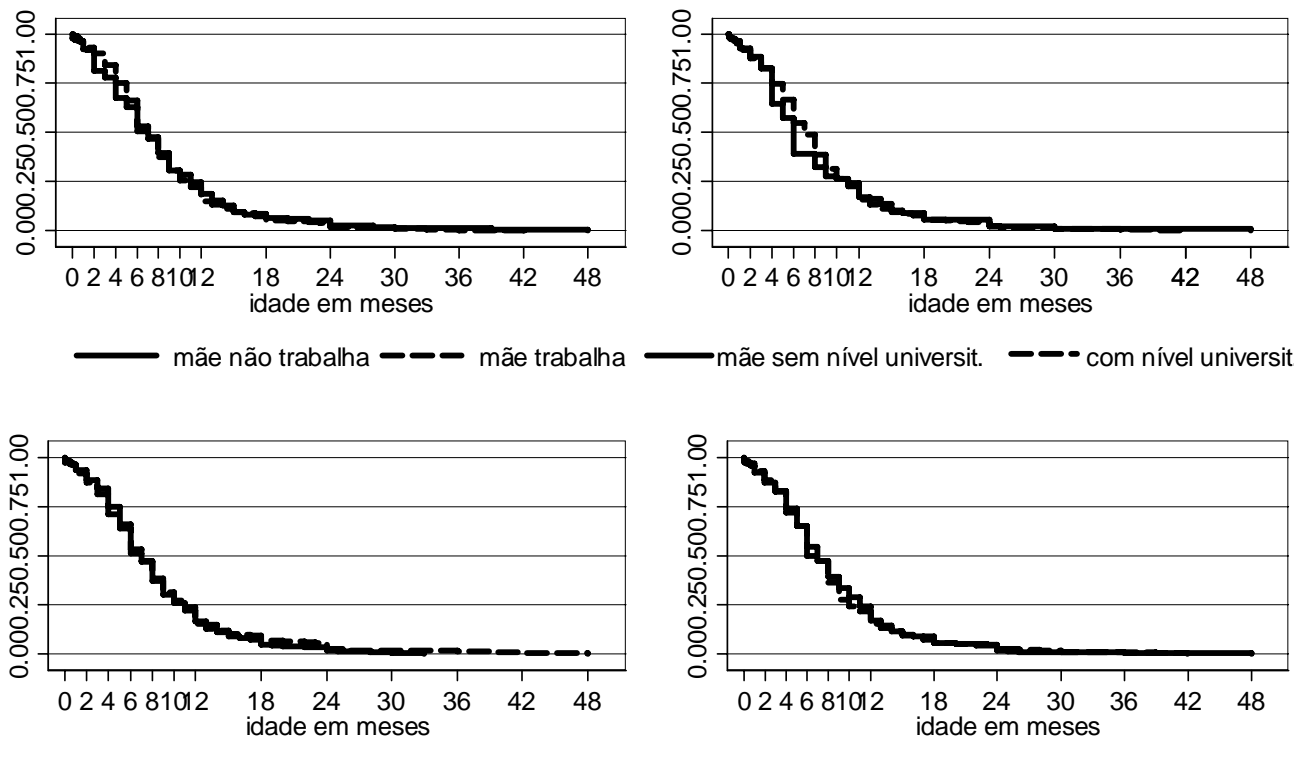

mãe com 35 anos ou menos - - -com mais de 35

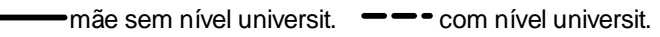

Tabela 2: Resultados da análise de regressão múltipla para variáveis maternas e sexo da criança. São Paulo, SP. 2004/2005.

\begin{tabular}{|c|c|c|c|c|c|c|}
\hline \multirow[t]{2}{*}{ Modelo } & \multicolumn{3}{|c|}{ AME } & \multicolumn{3}{|c|}{$\mathbf{A M}$} \\
\hline & OR & $\mathbf{p}$ & IC95\% & OR & p & IC95\% \\
\hline \multicolumn{7}{|l|}{ Trabalho materno } \\
\hline Trabalha fora & 1 & - & - & 1 & - & - \\
\hline Não trabalha & 0,96 & 0,70 & {$[0,79-1,18]$} & 1,03 & 0,76 & {$[0,85-1,25]$} \\
\hline \multicolumn{7}{|l|}{$\overline{\text { Escolaridade materna }}$} \\
\hline Ensino Fundamental + Médio & 1 & - & - & 1 & - & - \\
\hline Ensino Superior & 1,00 & 0,98 & {$[0,79-1,27]$} & 0,97 & 0,81 & {$[0,77-1,23]$} \\
\hline \multicolumn{7}{|l|}{ Idade materna } \\
\hline$\leq 35$ anos & 1 & - & - & 1 & - & - \\
\hline$>35$ anos & 1,14 & 0,14 & {$[0,96-1,34]$} & 0,95 & 0,56 & {$[0,80-1,13]$} \\
\hline \multicolumn{7}{|l|}{ Sexo da criança } \\
\hline Masculino & 1 & - & - & 1 & - & - \\
\hline Feminino & 0,98 & 0,87 & {$[0,83-1,16]$} & 1,02 & 0,82 & {$[0,86-1,21]$} \\
\hline
\end{tabular}

As figuras 2 e 3, completadas pela tabela 2, mostram não haver associação entre as variáveis estudadas e tempo de duração das duas formas de aleitamento. 


\section{Discussão}

A Organização Mundial de Saúde recomenda que a criança seja amamentada até o sexto mês de vida de forma exclusiva, e que a amamentação deve ser mantida por pelo menos dois anos ${ }^{1}$.

Apesar dessa recomendação e da maior difusão do conhecimento de que o leite materno, oferecido de forma exclusiva, atende as necessidades de energia e nutrientes do lactente até cerca de 6 meses de vida, verifica-se que a sua interrupção continua sendo precoce. Neste estudo observou-se que $78,6 \%$ das crianças estudadas deixaram de ser amamentadas exclusivamente antes dos 6 meses de vida e que apenas $24,1 \%$ das crianças mamaram por período superior a 1 ano de vida.

Nos últimos anos, como é de conhecimento dos pesquisadores da área de saúde, devido à importância e benefício do leite materno para a criança nos primeiros meses de vida, muitos estudos foram realizados para verificar prevalência, freqüência e duração da amamentação.

Em 1993, Gigante et al, ${ }^{13}$ verificaram na cidade de Pelotas, RS, mediana de amamentação de 3 meses. Na mesma cidade, em 2002/2003, Mascarenhas et al, ${ }^{14}$ com o objetivo de determinar a prevalência de aleitamento materno exclusivo justamente aos 3 meses de idade, observaram que de 940 lactentes, apenas 39\% ainda recebiam aleitamento materno exclusivo. Volpini \& Moura ${ }^{15} \mathrm{com}$ objetivo de investigar as características do desmame precoce no distrito Noroeste da cidade de Campinas, em 385 crianças, verificaram que $63,6 \%$ foram desmamadas precocemente, ou seja, antes dos seis meses de idade.

Em estudo com objetivo de descrever a situação da amamentação e identificar fatores associados ao desmame, em municípios do estado de São Paulo, foi observado que o aleita- mento materno nos primeiros 4 meses de vida raramente alcançou índices superiores a 30\%, sendo fator de risco para essa situação a baixa escolaridade materna, a primiparidade e a maternidade precoce. ${ }^{6}$

Em estudo realizado por Vieira et al ${ }^{16}$ com o objetivo de verificar a diferença de amamentação entre filhos de mães adolescentes e filhos de mães adultas, foi observado que, em relação ao aleitamento materno exclusivo, os dois grupos mantiveram mediana de 3 meses de duração, mostrando que a idade materna não influenciou na amamentação exclusiva. Já, em relação ao aleitamento materno, observaram que com 1 ano de idade, $35,3 \%$ e $28,5 \%$ dos filhos de mães adolescentes e adultas, respectivamente, continuavam sendo amamentados. Este resultado é interessante, pois mostra que mães adolescentes amamentam mais seus filhos do que mães adultas porem, não atribuem qualquer explicação a este fato.

Bueno et $\mathrm{al}^{17}$ ao descrever a prática do aleitamento materno de crianças nascidas em hospital universitário em São Paulo, e identificar fatores associados com a duração do aleitamento materno e aleitamento materno exclusivo, observaram que idade e escolaridade da mãe tiveram associação estatisticamente significante, com a duração apenas do aleitamento materno exclusivo. Segundo Hammer et $a l^{18}$, o conhecimento da idade materna é muito importante, entre outros fatores, pois muitos estudos a correlacionam com o tempo de desmame e com a idade de introdução de alimentos sólidos.

Neste estudo, a mediana e a prevalência de aleitamento materno exclusivo foram maiores que as verificadas nos estudos citados. Em relação à idade materna, observa-se que 47,2\% das mães estudadas tinham idade acima de 35 anos, mostrando uma população de mães adultas, não sendo observada associação estatisticamente significante entre duração do aleitamento materno e idade materna. 
Em relação à escolaridade, observou-se neste estudo, maior proporção de mães com nível universitário (84,6\%) mostrando uma população homogênea em relação a esta variável, o que de certa forma pode explicar a não associação com tempo de aleitamento materno exclusivo e aleitamento materno. Em outro estudo ${ }^{14}$, também não foi verificada associação estatisticamente significante entre interrupção do aleitamento materno exclusivo, antes do terceiro mês de vida, e escolaridade materna.

Diferentemente do encontrado neste estudo, Volpini \& Moura ${ }^{15}$ mostram que o tempo de estudo materno se associou ao desmame precoce. Mães com menor tempo de estudo tendiam a desmamar antes do seis meses $(p=0,04)$, e o risco de desmame precoce de uma mãe que estudou menos de cinco anos foi o dobro daquela que estudou 8 anos ou mais. Já

\section{REFERÊNCIAS}

1. World Health Organization. The optimal duration of exclusive breastfeeding: a systematic review. Geneva: WHO; 2001.

2- World Health Organization. Indicators for assessing breastfeeding practices. Update. Programme Control Diarrhoeal. Dis 1992; (10):1-4.

3. Krammer MS, Chalmers B, Hodnett ED, Sevkovskaya Z, Dzikovich I, Shapino S et al. Promotion of breastfeeding intervention trial (PROBIT): a randomized trial in the republic of Belarus. JAMA. 2001;285:416-20.

4. Venâncio SL, Monteiro CA. A evolução da prática da amamentação nas décadas de 70 e 80. Rev Bras Epidemiologia. 1998; (1):40-9. a variável trabalho materno apresentou resultado semelhante ao encontrado neste estudo, não se associando ao desmame precoce.

Ao contrário do verificado neste estudo, algumas pesquisas mostram associação estatisticamente significante entre sexo da criança e duração de aleitamento materno ${ }^{19}$. Segundo Guttierez et al, ${ }^{20}$ a manutenção do aleitamento materno depende, entre outros fatores, das informações que os profissionais de saúde passam para as mães.

O presente estudo não detectou associação da duração do aleitamento materno e aleitamento materno exclusivo, porém verificouse que cerca de $80 \%$ das crianças deixaram de ser amamentadas exclusivamente antes dos seis meses de vida, o que mostra a necessidade de continuar o desenvolvimento de ações para incentivo e apoio à amamentação.
5. Leão MM, Coitinho DC, Recine E, Costa LAL, Lacerda AJ. O perfil do aleitamento no Brasil. In: Fundação IBGE. Perfil estatístico de crianças e mães no Brasil. Rio de Janeiro. 1992, 97-109.

6. Venâncio SL, Escuder MML, Kitoko P, Rea MF, Monteiro CA. Freqüências e determinantes do aleitamento materno em municípios de Estado de São Paulo. Rev Saúde Pública. 2002; 36(3):313-8.

7. Ministério da Saúde. Prevalência de aleitamento materno nas capitais brasileiras e no Distrito Federal. Brasilia. 2001. Relatório preliminar.

8. Silveira FJF, Lamounier JA. Prevalência do aleitamento materno e praticas de alimentação complementar em crianças com até 24 meses de idade da região do Alto Jequitinhonha, Minas Gerais. Rev Nutr. 2004; 17(4):437-447. 
9. Camilo DF, Carvalho RVB, Oliveira EF, Moura EC. Prevalência da amamentação em crianças menores de dois anos vacinadas nos centros de saúde escola. Rev Nutr. 2004;17(1):29-36.

10. Ministério da Saúde. II Pesquisa de prevalência de aleitamento materno nas Capitais Brasileiras e Distrito Federal. http://portal.saude.gov.br. 13 de novembro de 2009.

11. Vituri SC, de Brito ASJ. Prevalência do aleitamento materno em crianças até o sexto mês de idade na cidade de Maringá, estado do Paraná, Brasil. Acta Sci Health Sci. 2003;25:141-6.

12. Oliveira LPM, Assis AMO, Pinheiro SMC, Prado MS, Barreto ML. Alimentação complementar nos primeiros dois anos de vida. Rev Nutr. 2005; 18:459-69.

13. Gigante DP, Victora CG, Barros FC. Nutrição materna e duração da amamentação em uma coorte de nascimento de Pelotas, RS. Rev Saúde Pública. 2000; 34(3):259-65

14. Mascarenhas MLW, Albernaz EP, Silva MB, Silveira RB. Prevalence of exclusive breastfeeding and its determiners in the first 3 months of life in South of Brazil. J Pediatr. 2006; 82(4):289-94.
15. Volpini CCA, Moura EC. Determinantes do desmame precoce no distrito noroeste de Campinas. Rev Nutr. 2005; 18(3):311-319.

16. Vieira MLF, Silva JLCP, Filho AAB. A amamentação e a alimentação complementar de filhos de mães adolescentes são diferentes das de filhos de mães adultas? J Pediatr. 2003; 79(4):317-24.

17. Bueno MB, Souza JMP, Souza SB, Paz SMRS, Gimeno SGA, Siqueira AAF. Riscos associados ao processo de desmame entre crianças nascidas em hospital universitário de São Paulo, entre 1998 e 1999: estudo de coorte prospectivo do primeiro ano de vida. Cad Saúde Pública. 2003; 19(5):1453-1460.

18. Hammer LD, Bryson S, Agrias WS. Development of feeding practices during the first 5 years of life. Am J of Clin Nutr. 1999; 153:189-94.

19. Pérez-Escamilla R, Lutter C, Segall AM, Rivera A, Trevino-Siller S, Sanghvi T. Exclusive breastfeeding duration is associated with attitudinal, socioeconomic and biocultural determinants in three latin american countries. J Nutrition.1995; 125:2972-84.

20. Gutierrez L, Delgado SE, Costa, AP. Caracterização do uso da técnica do copo em UTI neonatal de um hospital público. Rev Bras Crescimento Desenvolv Hum. 2006; 16(1): 22-31.

Recebido em 12 de julho de 2009. Modificado em 02 de agosto de 2009. Aceito em 22 de setembro de 2009. 